\title{
Avanzando en la gestión y seguridad terapéutica: recuento de comprimidos
}

Rocío Coto Moreno - Ángeles Laviana Fernández - Azucena Pereda González - Ruth Álvarez Oviedo

- Covadonga Díaz García - Patricia Esteban Hernández - Carmen Blanco Suárez - Gloria González

Suárez - Beatriz Diez Ojea - Manuel Ángel Alonso Álvarez -

Unidad de Hemodiálisis del Hospital Valle del Nalón

\section{Sra. Directora:}

Se define la cumplimentación terapéutica como la medida en que la conducta del paciente en relación con la toma de medicación, seguimiento de una dieta o modificación de su estilo de vida, coincide con las indicaciones dadas por los profesionales sanitarios. El incumplimiento, repercute negativamente en la calidad de vida y aumenta los costes. Esta falta de adherencia terapéutica se relaciona con múltiples factores en relación con el paciente, el profesional, el sistema sanitario, la enfermedad y los fármacos.

Revisando las taxonomías enfermeras NANDA, NIC, NOC comprobamos que el uso seguro de la medicación propuesto implica un conocimiento mucho más ambicioso que el que transmitía la hoja de tratamiento que se utilizaba hasta ese momento ya que es necesario garantizar la seguridad y el control sobre el tratamiento de los pacientes. Ante las preguntas: ¿Cómo puede influir enfermería en los pacientes para conseguir conductas de cumplimiento? Y ¿Cómo pasar de hábitos insanos a conductas positivas? nos planteamos mejorar la seguridad terapéutica y gestionar los tratamientos farmacológicos de los pacientes cambiando el mecanismo de transmisión de la información además de proporcionar

\author{
Correspondencia: \\ Rocío Coto Moreno \\ Unidad de Hemodiálisis \\ Hospital Valle del Nalón \\ Polígono de Riaño s/n \\ 33920 Riaño (Langreo) \\ carmenblansu@gmail.com
}

educación y conocimiento individualizados. A tal fin se realizó una encuesta sobre la pauta terapéutica y evaluación de conocimientos a 41 pacientes. Se excluyó de la evaluación de conocimientos a 7 pacientes que presentan deterioro cognitivo $y / 0$ incapacidad para el aprendizaje.

Se creó una aplicación informática para la gestión de los tratamientos personalizados que aportara información adicional y que ayudara a mejorar y reforzar los conocimientos de los pacientes. Para facilitar el control del cumplimiento al personal de enfermería la aplicación permite realizar el recuento de comprimidos informatizado.

Se diseñó una "hoja de ayuda" para que los pacientes soliciten los medicamentos prescritos y se realizan las intervenciones de seguridad que el personal de enfermería estime oportunas incluyendo el "recuento de comprimidos por blister". Se elaboró un "dossier terapéutico" para mejorar el aprendizaje de los pacientes de la nueva hoja de tratamiento que consta de varios apartados: recomendaciones importantes, consumo responsable, uso racional de antibióticos, almacenamiento, caducidad y reciclaje.

Se realiza anualmente una entrevista utilizando los test del Cumplimiento Haynes-Sackett (HS) y Morinsky Green (MG) a los que se incorpora la efectividad y accesibilidad del personal de la unidad y la persona responsable de la preparación de la medicación: el paciente, el cuidador principal, un familiar...

Disponemos de una hoja de valoración de conocimientos (figura 1) con las intervenciones de enfermería nece- 
sarias según la taxonomía NIC para 4 indicadores de los propuestos por la taxonomía NOC:

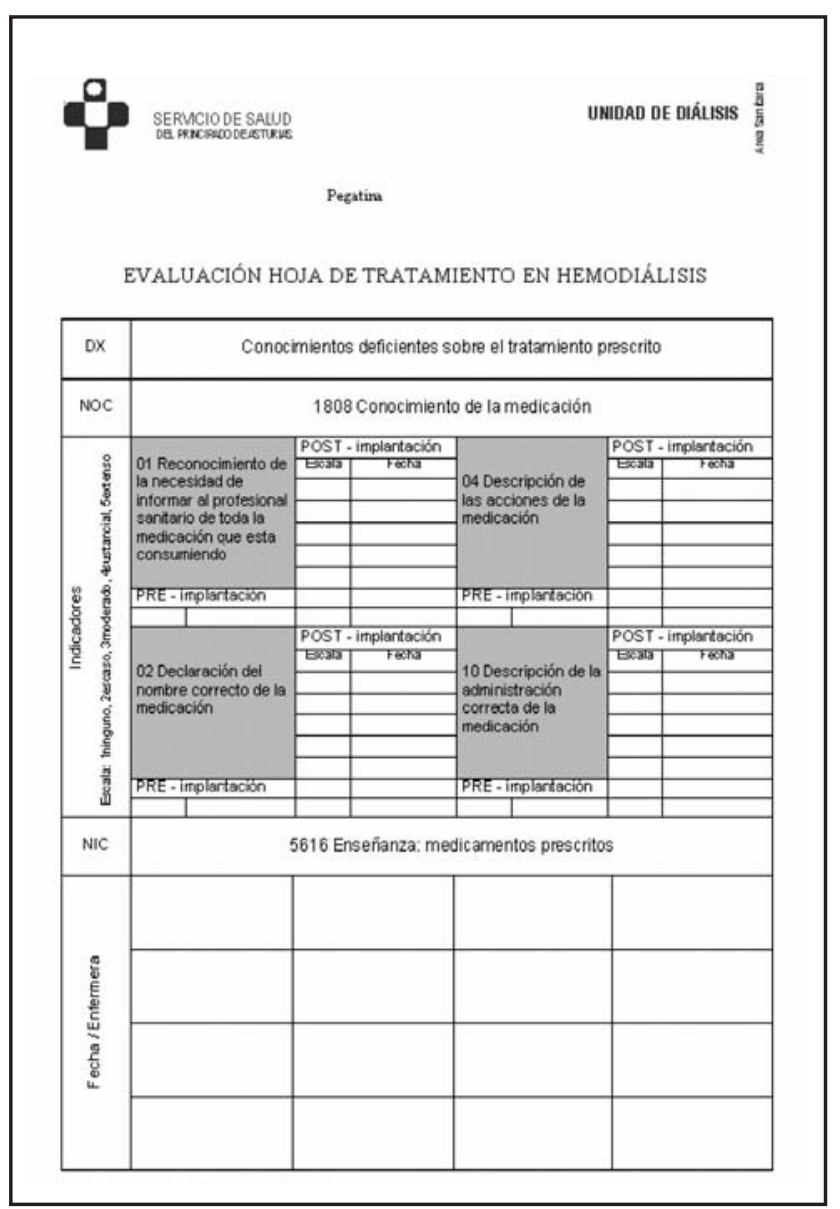

Figura 1. Hoja de evaluación de conocimientos

- Indicador 01: Reconocimiento de la necesidad de informar al profesional sanitario de toda la medicación que está consumiendo

- Indicador 02: Declaración del nombre correcto de la medicación

- Indicador 04: Descripción de las acciones de la medicación

- Indicador 010: Descripción de la administración correcta de la medicación

Se realizó la valoración de conocimientos de los pacientes sobre su tratamiento terapéutico pre-implantación de la nueva hoja de tratamiento y una vez realizada la educación con ésta se valoran los conocimientos del paciente al menos 1 vez al año.

Nuestros resultados demuestran que se ha modificado la hoja de tratamiento haciendo que la complejidad de sus tratamientos de un vuelco y se convierta en una labor diaria entretenida, instructiva y más sencilla de realizar e intentando que la información entre por los ojos de quien la mira.

- En el año 2007 se encuestaron 29 pacientes (16 hombres y 13 mujeres con una edad media de 68 años) que tomaban 97 comprimidos semanales de media, de estos 15 pacientes preparan la medicación y 14 reciben ayuda del cuidador. El 75,86\% de los pacientes entienden la hoja de tratamiento que reciben. La efectividad y accesibilidad es del $100 \%$.

- En el año 2008 se inicio el pilotaje con el nuevo formato de hoja de tratamiento y se encuestaron 31 pacientes (17 hombres y 14 mujeres) con una media de edad de 70 años que tomaban 102 comprimidos semanales de media. Quince pacientes preparan la medicación y 16 pacientes reciben ayuda del cuidador. El $73,91 \%$ entienden la hoja de tratamiento que reciben. El $100 \%$ de pacientes responden entender el nuevo formato. La efectividad es del $100 \%$ y la accesibilidad es del 97\%. Según el test de HaynesSackett el $100 \%$ de los pacientes son cumplidores y según el test de Morinsky Green lo son un 77,4\%.

- En el año 2009 (del 1 de enero a 31 de marzo) se encuestaron 30 pacientes (19 hombres y 11 mujeres). La media de edad es de 68 años y toman 90 comprimidos de media semanales; 18 pacientes preparan la medicación, 11 reciben ayuda del cuidador y 1 colabora con el cuidador en la preparación. El $79,3 \%$ entienden la hoja de tratamiento que reciben. La efectividad y accesibilidad es del 100\%. Según el test de Haynes Sackett el 100\% de los pacientes son cumplidores y según el test de Morinsky Green lo son un $72,4 \%$ (figura 2 ).

Se han realizado 120 intervenciones NIC: "Enseñanza: medicamentos prescritos", 20 recuentos de comprimidos por blister y 469 intervenciones de seguridad. EI recuento de comprimidos informatizado valora periódicamente la adherencia al tratamiento y nos permiten gestionar adecuadamente los recursos sanitarios. Las intervenciones de enfermería individualizadas refuerzan 
PRE-EDUCACIÓN

- POST-EDUC.: $1^{\mathrm{a}}$ EVAL.

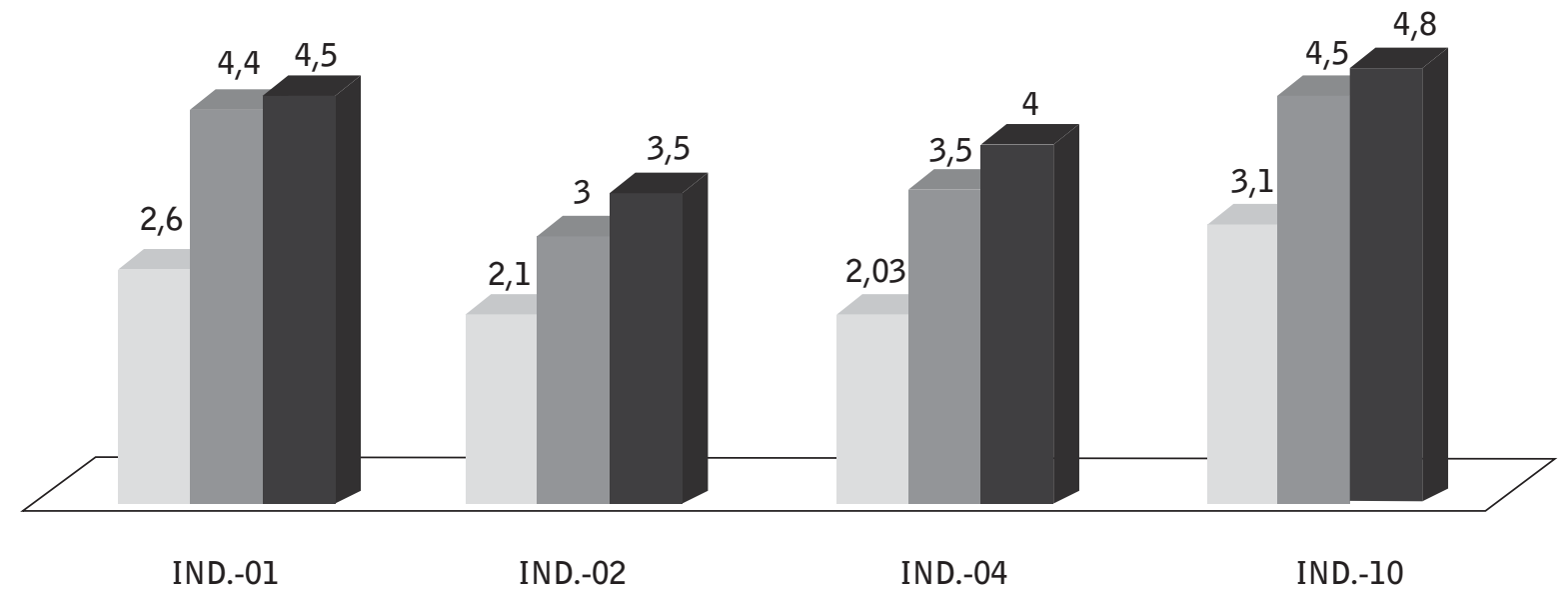

Figura 2: Resultados en la adquisición de conocimientos de las distintas valoraciones

conductas positivas y ayudan a modificar conductas de no cumplimiento.

Mediante la modificación del formato y contenido de la hoja de tratamiento, podemos transmitir la misma información de manera sencilla y eficaz, ampliándola para mejorar los conocimientos que los pacientes tienen de su tratamiento y conseguir conductas de cumplimiento (figura 3). Con el recuento de comprimidos se consigue un seguimiento exhaustivo del tratamiento, una gestión adecuada y el refuerzo de la seguridad del paciente. Creemos que la mejora de conocimien-

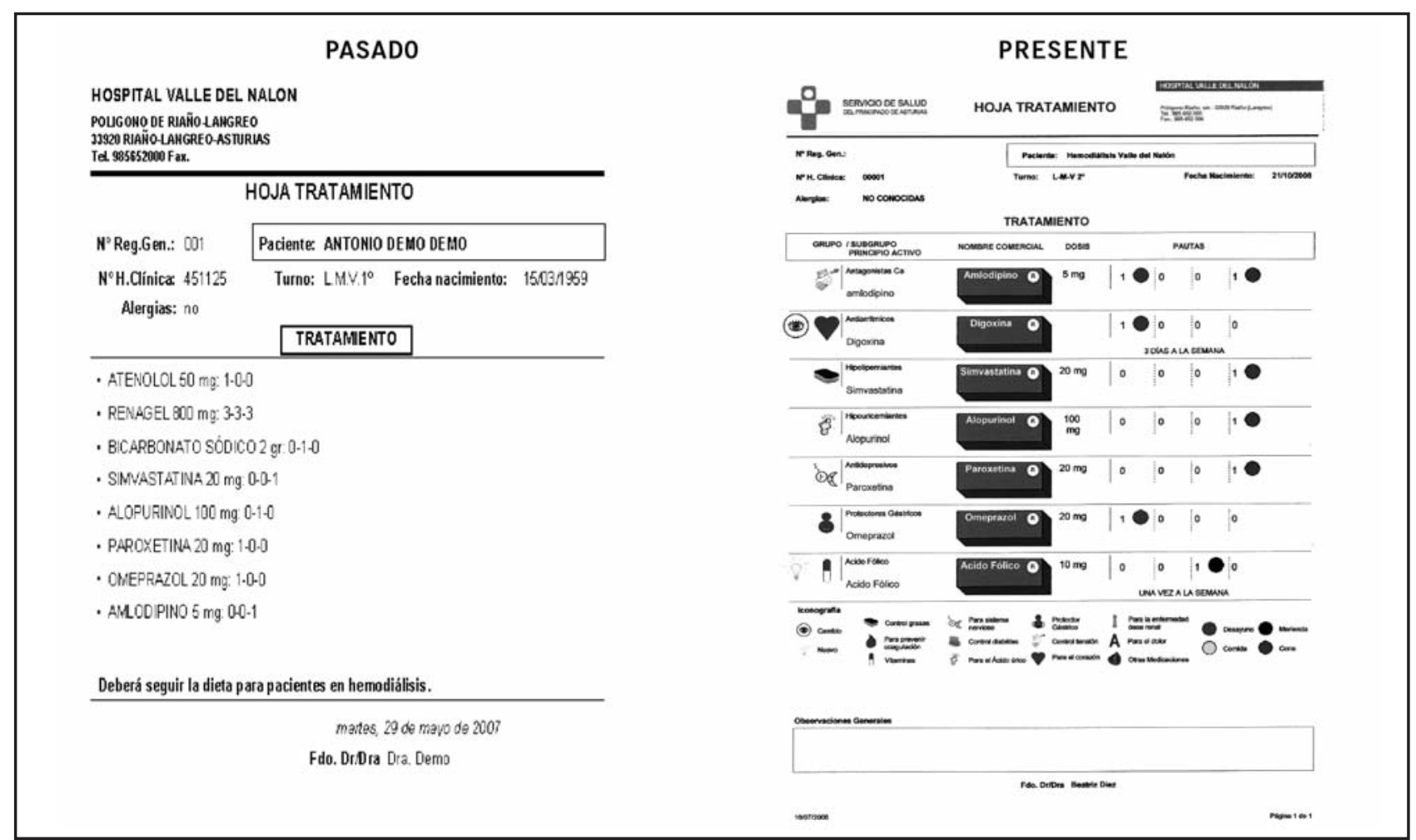

Figura 3: Pasado y presente en las hojas de tratamiento. 
tos mediante la educación sanitaria permite avanzar en seguridad en los pacientes con polifarmacia. EI compromiso adquirido por el personal de enfermería con el control terapéutico supone un gran avance en seguridad del paciente.

¿Qué nos planteamos de aquí en adelante?, seguir por el camino que hemos empezado y establecer como objetivo de enfermería 2010, la conciliación terapéutica con la familia/cuidador principal de aquellos pacientes con incapacidad para el aprendizaje y de otros que se estime necesario, pues consideramos importante valorar los conocimientos al familiar responsable de gestionar la medicación del paciente.

\section{Bibliografía}

- Diagnósticos enfermeros: Definiciones y Clasificación 2001-2002 NANDA internacional. Harcourt. 2001.

- Jhonson M, Bulechek G, McCloskey Dochterman J, Maas M, Moorhead S. Interrelaciones NANDA-NOCNIC Diagnósticos enfermeros, Resultados e intervenciones. $2^{\mathrm{a}}$ ed. Elselvier. 2007.

- Jhonson M, Bulechek G, McCloskey Dochterman J, Maas M, Moorhead S. Diagnósticos enfermeros, Resultados e intervenciones. Elselvier. 2004. 